The sociodemographic context of observed solitary and social smoking behaviours using a behavioural ecological approach

\author{
Felix Yong Peng Why \\ University of Hull \\ Anna Undarwati \\ University of Hull
}

Siti Nuzulia

University of Hull

Contact Information:

Felix Yong Peng Why

University of Hull

Cottingham Road,

HU6 7RX,

Hull, UK.

Acknowledgements

We would like to thank Rebecca Magri for her assistance in data collection.

Conflict of Interest statement

There is no conflict of interest to be declared. 


\begin{abstract}
This study used a behavioural ecological approach by observing whether solitary and social smoking varied as a function of gender and stress. In sample $1(N=414)$, the result was consistent with the Tend-and-Befriend Hypothesis in that more female smokers were observed to engage in social smoking during high stress. When the number of smokers observed by stress condition was controlled for in sample $2(N=587)$, this effect was non-significant. Effect sizes were small for both samples. Discrepancies with previous research suggests that self-reported data might over-estimate the interaction of various psychosocial factors on smoking behaviours.
\end{abstract}

Keywords: smoking, stress, coping, social smoking, gender, social support 


\section{The sociodemographic context of observed solitary and social smoking behaviours using a behavioural ecological approach}

Smoking can be done alone (solitary smoking) or in the company of others (social smoking). Smokers have reported a number of motives for smoking such as coping with stress and socializing with others (Delaney, MacGregor, \& Amos, 2018). Hence, social smoking (cf. solitary smoking), can be regarded as the smoker engaging in social affiliation with others while using tobacco. Social smoking, as a form of social affiliative behaviour, is reported by smokers to be elicited particularly during psychological stress exposure (Nichter, 2015). The prevalence of social smoking based on selfreported data have ranged from $30 \%$ to $70 \%$ (Berg, \& Lin, 2019). Social smoking is an important smoking behaviour because of its reported high prevalence and health consequence when used as a form of coping strategy for stress: even when social smoking is done intermittently (e.g., only smoke at social events), it still carries significant health risks (Inoue-Choi, McNeel, Hartge, \& Caporaso, 2019). In addition, social smokers tend not to consider themselves as 'smokers' and hence, smoking cessation services are unlikely to target social smokers effectively because social smokers are less likely to seek treatment (Berg, Lin, White \& Alfonso-Barry, 2017).

Taylor et al.'s (2000) Tend-and-Befriend hypothesis postulates that social affiliative behaviours are not only elicited during periods of psychological stress but this elicitation is stronger among women. In this manuscript, we attempt to test the Tend-and-Befriend hypothesis for social and solitary smoking. If social smokers report using smoking as a form of social affiliation activity, then this Hypothesis can be tested for this behaviour. However, the complex evidence (e.g., neuroendocrine pathways, behavioural data from human and non-human species) reviewed by Taylor et al. (2000) were circumstantial. For example, most evidence reviewed by these authors point to the main effects of stress or gender but do not specifically test the interaction of these factors in predicting social affiliative behaviours. The review also does not cover the neuroendocrine pathways (e.g., testosterone, cortisol) that has been found to regulate social affiliative behaviours among males, which would not support gender differences in social affiliative behaviours. To our knowledge, the only study that was formulated to test this hypothesis directly was reported by Bodenmann and his colleagues (Bodenmann et al., 2015), which analysed the verbal communication between partners engaging in a conversation in a non-smoking context. They found that men's, not women's, positive (e.g., verbal statements that comfort the partner) and negative (e.g., verbal statements that dismiss the partner's experience) social support verbal communication varied more strongly as a function of stress. Our study aims to address the paucity of empirical evidence for this Hypothesis using a naturalistic systematic observational approach for smoking behaviours.

In addition, the majority of research on social smoking have relied on self-reported data. For example, Moran et al. (2004) defined social smoking as participants who reported mainly smoking with other people in the last 30 days. There is evidence that smoking is an automatic habitual behaviour where people who smoke might have poor insight into the motives and causes for their smoking (Hagger, Rebar, Mullan, Lipp, \& Chatzisarantis, 2015); self-reported smoking has often been found to be inconsistent with behavioural data such as puffing behaviour (Krebs, Chen, Zhu, Sun, Liao, Stennett, \& Muscat, 2016). In this study, we examine the prevalence and sociodemographic context of solitary and social smoking using a behavioural ecological approach. The academic setting presents a suitable environment to conduct this research because of its predictable cycle of high-low stress periods (e.g., Pitt, Oprescu, Tapia, \& Gray, 2018). Using this predictable stress cycle, we observe the sociodemographic profile of individuals who engage in solitary or social smoking behaviours. Based on previous published research, we examine whether the prevalence of social 
smoking would be higher among women during high stress as put forward by the Tend-and-Befriend Hypothesis (Taylor et al., 2000).

\section{Method}

Participants. Our target minimum sample size was 263 assuming a small-medium effect size of Cohen's $w=.20$, with the following parameters: alpha $=.05$, power $=.90, \mathrm{df}=1$. Two samples were acquired: Sample 1 consisted of 414 individuals ( 256 men, 158 women) while sample 2 consisted of 587 individuals ( 374 men, 213 women). Sample 1 employed interval recording (i.e., 10 observation sessions done between $1200 \mathrm{hr}$ to $1400 \mathrm{hr}$ on weekdays) while sample 2 employed event recording (i.e., observations of social/solitary smoking prevalence for an equal number of observees between the two stress periods). This is because there are likely to be more people observed smoking during high stress periods (Pitt et al., 2018). Hence, sample 2's observational strategy controls for this. A university campus was selected as the venue for this study. This study has been approved by the institutional review board and is a pre-registered study at the Open Science Framework (www.osf.io; doi: 10.17605/OSF.IO/7GREA).

Materials. Ambient temperature influences smoking behaviours and was measured using a handheld digital meter (Benetech GM1361) which has an accuracy of $\pm 1^{\circ} \mathrm{C}$. Ambient temperate was assessed at the start of each observation session (Table 1).

$<$ INSERT TABLE 1 HERE>

Procedure. We identified designated observation areas on a university campus where people smoke. These areas were approximately $4 \mathrm{~m} \times 4 \mathrm{~m}$ in an outdoor area. Social smoking was defined as a person observed smoking with another person or in a group as reflected in previous research using questionnaires (e.g., Moran et al. 2004). In sample 1, we used interval sampling via recording the people observed entering these areas to smoke. In sample 2, we used event sampling by observing 294 smokers during the low stress period and 293 smokers during the high stress period (i.e., number of observed individuals was similar across the two stress periods). Our observations for both samples were done between $1200 \mathrm{hrs}$ and $1400 \mathrm{hrs}$ on randomly selected weekdays. The observations for samples 1 and 2 were done over the same months but on different days. For each observed smoker, we recorded the gender of the smoker and whether the smoker smoked alone or in a group. If a person smoked in a group, the first person who entered the designated area to smoke was identified as the observee. We defined group membership of a smoker in the following observed behaviours: (i) at least one other person accompanied the target observee at the designated area, and/or (ii) engaging in a conversation with the observee. All observers made their observations at least $2 \mathrm{~m}$ from these designated areas to avoid tobacco smoke exposure. In line with previous research (e.g., Pitt et al., 2018), we defined high stress as periods when examinations/formative assessments occur during an academic calendar (e.g., January), while low stress was defined by the academic teaching weeks where the occurrence of formal assessments was low (e.g., November). High and low stress observation sessions were picked in months as close to each other as possible to minimise seasonal differences in ambient temperature. Observations were done between November 2018 to February 2019. 
Alpha level is set at .05 . We tested the Tend-and-Befriend Hypothesis in a 3 way contingency table (Gender X Stress X Solitary/Social Smoking behaviour) using Cochran test of conditional independence and Mantel-Haenszel test of conditional independence. The latter is a more conservative statistical test. In addition, we used the Mantel-Haenszel Common Odds Ratio estimate to assess whether the Gender X Solitary/Social Smoking behaviour 2 way interaction is significant after controlling for the effects of Stress.

Sample 1. We observed more male smokers and more smokers during the high stress period. We used the Cochran test of conditional independence and Mantel-Haenszel test of conditional independence to test the 3 way contingency table (Stress by Gender X Social Smoking Status; Table 1). Gender $X$ Social Smoking by Stress interaction was of borderline significance: Cochran test of conditional independence $\chi^{2}(1, n=414)=4.19, p=.04, \Phi=.10$, Mantel-Haenszel test of conditional independence $\chi^{2}(1, n=414)=3.77, p=.05, \Phi=.10$. According to Cohen (1988), $\Phi=.10$ is considered a 'small' effect size. The trend of the observations is consistent with the Tend-andBefriend Hypothesis: more female smokers were observed engaging in social smoking during high stress than male smokers. Controlling for stress periods, there was a gender difference in social and solitary smoking: Mantel-Haenszel Common Odds Ratio estimate was significant, $\mathrm{OR}=1.52, p=.04$, $95 \% \mathrm{Cl}[2.26,1.02]$. When controlling for stress, female smokers were significantly more likely to be observed engaging in social smoking (54.4\%) than male smokers (44.1\%).

Sample 2. The Gender by Social Smoking by Stress interaction was non-significant: Cochran test of conditional independence $\chi^{2}(1, n=587)=3.03, p=.08, \Phi=.07$, Mantel-Haenszel test of conditional independence $\chi^{2}(1, n=587)=2.72, p=.10, \Phi=.07$. Controlling for stress, the Gender by Social Smoking was also non-significant for this sample, Mantel-Haenszel Common Odds Ratio estimate was significant, $\mathrm{OR}=1.35, p=.08,95 \% \mathrm{Cl}[1.91,0.96]$.

\section{Discussion}

We believe our study is the only study that has examined the psychosocial context of solitary and social smoking using a behavioural ecological approach. Our results provided inconsistent support for the Tend-and-Befriend Hypothesis (Taylor et al., 2000) with relation to observed social smoking behaviours. In sample 1, our results were consistent with this Hypothesis: female smokers were marginally more likely to engage in social smoking than male smokers during high stress periods. However, this gender difference in social smoking was also present for both low and high stress periods. Results from sample 2 indicate that a significant factor in the borderline significant results obtained in sample 1 was partly due to the sample size differences obtained between high and low stress periods. Regardless of its statistical significance, we found that the effect sizes associated with this Hypothesis were small.

Previous research found that college students reported that they were more likely to engage in social smoking during periods of high stress as a coping strategy (Nichter, 2015). Our observational data did not support this finding: in both samples, the prevalence of social smoking, a specific form of smoking observed in our samples, did not increase as a function of stress. The increase in social smoking observed in sample 1 during high stress period is due to the higher number of people observed (solitary and social) smoking during this period. When this sample size difference was controlled for, social smoking had a stable prevalence across the two stress levels. The prevalence of social smoking that we have observed in our two samples were lower when compared to that derived from self-reported data: when compared to what previous research has have found (Berg \& 
Lin, 2019), self-reported data over-estimated the prevalence of social smoking by about $10 \%$ to $30 \%$ when compared to our observations. Our observational data was congruent with national survey data that reported a higher prevalence of smoking behaviours among men when compared to women (http://ons.gov.uk) as well higher smoking likelihood during high stress (Pitt et al., 2018).

The relative contextual stability of social smoking, a specific form of smoking behaviour, found in our two samples suggests that people's tendency to engage in social or solitary smoking is likely to be an automatic and stable behaviour rather than one that fluctuates significantly with changes in psychosocial context. Such habit stability could be due to personal preference towards solitary/social smoking, the half-life of nicotine and its association with craving and withdrawal symptoms (e.g., every 2 hours) or at specific regular events (e.g., meal times; Dunbar, Scharf, Kirchner, \& Shiffman, 2010). Our results suggest the need to validate questionnaire-based findings about the social and psychological motives surrounding smoking behaviours using other non-selfreported data. The discrepancies between self-reported smoking behaviours and observed smoking behaviours suggest that smokers might have poor psychological insight about the causes and motives that influence their habitual behaviours such as smoking (Hagger, Rebar, Mullan, Lipp, \& Chatzisarantis, 2015).

Our study has a few limitations. Firstly, our results do not reject the proposition that there are significant main effects for stress and gender that influence smoking behaviours. On the contrary, our data is consistent with the main effects of stress and gender on smoking behaviours. We did not, however, find consistent nor strong evidence to indicate that people's tendency to engage in social smoking varied as a function of the interactive effects of gender and stress levels. In other words, more men smoke, more people smoke when under stress, but women do not necessarily smoke more with others when under stress. Secondly, our operationalisation of social smoking did not include this behaviour as observed in other venues (e.g., at parties). The higher prevalence of social smoking reported in previous research might be due to smokers using a specific salient context where socialising is a core activity (e.g., at parties) as a reference point to overestimate their average social smoking frequency (i.e., confirmatory bias). Thirdly, our observations did not include whether a social smoker smokes regularly or not; our studies were anonymous observations and did not collect within-person smoking behaviour data. Lastly, we classified the gender of our observees based on physical appearance and gender identity could not be assessed via observational methods. However, a meta-analysis found that the prevalence of discrepancies between sex and gender identity (e.g., gender dysphoria) is low (i.e., 4.6/100000; Arcelus, Bouman, Van Den Noortgate, Claes, \& Witcomb, 2015) and hence, gender misclassification is unlikely to affect our results significantly. Future research might wish to apply our methods to address limitations. In addition, though ambient temperature was similar across the stress periods within each sample, future studies could conduct observations across a wider ambient temperature range and include them into the statistical model.

In conclusion, our studies found that the effects for the Tend-and-Befriend Hypothesis among female smokers are inconsistent and weak. Significant result for this Hypothesis could partly be explained by the sample size difference observed for smoking between high and low stress periods. The patterns of observed solitary and social smoking behaviours are also discrepant with previous research based on self-reported data. Our naturalistic systematic observation of smoking behaviours suggests caution in concluding smoking behaviours based on what smokers' report about the circumstances and motivations influencing their smoking habits. That is, a smoker's psychological representation of their smoking behaviours might not be congruent with their actual smoking behaviours. 
Reference List

Arcelus, J., Bouman, W. P., Van Den Noortgate, W., Claes, L., \& Witcomb, G. (2015).

Systematic review and meta-analysis of prevalence studies in transsexualism. European Psychiatry, 30, 807-815. 10.1016/j.eurpsy.2015.04.005

Berg, M. B., Lin, L., White, M., \& Alfonso-Barry, J. (2017). Attitudinal and behavioral differences between cigarette users who do and do not identify as "smokers". Journal of American College Health, 65, 249-257. doi: 10.1080/07448481.2017.1312417

Berg, M. B., \& Lin, L. (2019). Occasionally stigmatized: how the frequency and context of use influence perceptions of intermittent smokers. Addiction Research \& Therapy, 27, 249-257. doi: $10.1080 / 16066359.2018 .1499897$

Bodenmann, G., Meuwly, N., Germann, J., Nussbeck, F. W., Heinrichs, M., \& Bradbury, T. N. (2015). Effects of Stress on the Social Support Provided by Men and Women in Intimate Relationships. Psychological Science, 26, 1584-1594. 10.1177/0956797615594616

Cohen, J. (1988). Statistical power analysis for the behavioral sciences. (2nd ed.) New Jersey: Lawrence Erlbaum.

Delaney, H., MacGregor, A., \& Amos, A. (2018) “Tell them you smoke, you'll get more breaks": a qualitative study of occupational and social contexts of young adult smoking in Scotland. BMJ Open, 8, e023951. doi: 10.1136/bmjopen-2018-023951

Dunbar, M. S., Scharf, D., Kirchner, T., \& Shiffman, S. (2010). Do smokers crave cigarettes in some smoking situations more than others? Situational correlates of craving when smoking. Nicotine \& Tobacco Research, 12, 226-234. 10.1093/ntr/ntp198 
Inoue-Choi, M., McNeel, T. S., Hartge, P., \& Caporaso, N. E. (2019). Non-Daily Cigarette Smokers: Mortality Risks in the U.S. American Journal of Preventive Medicine, 56, 27-37. doi: 10.1016/j.amepre.2018.06.025

Krebs, N. M., Chen, A., Zhu, J., Sun, D., Liao, J., Stennett, A. L., \&, Muscat, J. E. (2016). Comparison of Puff Volume With Cigarettes per Day in Predicting Nicotine Uptake Among Daily Smokers. American Journal of Epidemiology, 184, 48-57. doi: 10.1093/aje/kwv341

Nichter, M. (2015). Lighting Up: The Rise of Social Smoking on College Campuses. New York: New York University Press.

Hagger, M. S., Rebar, A. L., Mullan, B., Lipp, O. V., \& Chatzisarantis, N. L. D. (2015). The subjective experience of habit captured by self-report indexes may lead to inaccuracies in the measurement of habitual action. Health Psychology Review, 9, 296-302. doi:

$10.1080 / 17437199.2014 .959728$

Moran, S., Wechsler, H., \& Rigotti, N. A. (2004). Social smoking among US college students. Pediatrics, 114, 1028-1034. 10.1542/peds.2003-0558-L

Pitt, A., Oprescu, F., Tapia, G., \& Gray, M. (2018). An exploratory study of students' weekly stress levels and sources of stress during the semester. Active Learning in Higher Education, 19, 6175. doi: $10.1177 / 1469787417731194$

Taylor, S. E., lein, L. C., Lewis, B. P., ruenewald, T. L., Gurung, R. A., \& Updegraff, J. A. (2000). Biobehavioral responses to stress in females: tend-and-befriend, not fight-or-flight. Psychological Review, 107, 411-429. 10.1037/0033-295X.107.3.411 
Table 1

Number of observations (\%) by Gender, Social Smoking status, and Stress period

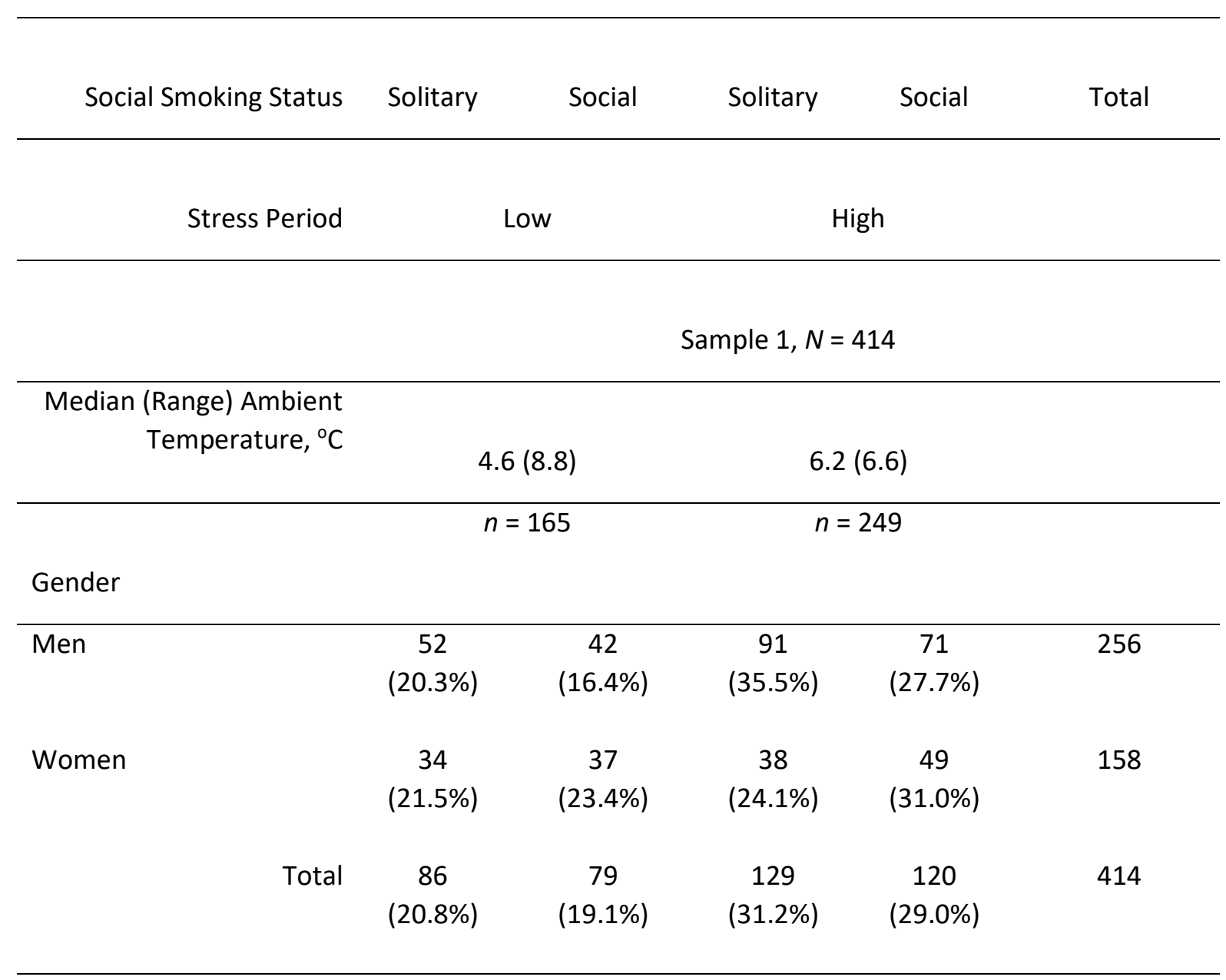

Sample $2, N=587$

\begin{tabular}{rcc}
\hline $\begin{array}{r}\text { Median (Range) Ambient } \\
\text { Temperature, }{ }^{\circ} \mathrm{C}\end{array}$ & $10.5(6.8)$ & $10.6(0.7)$ \\
\hline & $n=294^{*}$ & $n=293^{*}$
\end{tabular}

Gender

\begin{tabular}{|c|c|c|c|c|c|c|}
\hline Men & & $\begin{array}{c}119 \\
(31.8 \%)\end{array}$ & $\begin{array}{c}74 \\
(19.8 \%)\end{array}$ & $\begin{array}{c}114 \\
(30.5 \%)\end{array}$ & $\begin{array}{c}67 \\
(17.9 \%)\end{array}$ & 374 \\
\hline \multirow[t]{2}{*}{ Women } & & $\begin{array}{c}58 \\
(27.2 \%)\end{array}$ & $\begin{array}{c}43 \\
(20.2 \%)\end{array}$ & $\begin{array}{c}59 \\
(27.7 \%)\end{array}$ & $\begin{array}{c}53 \\
(24.9 \%)\end{array}$ & 213 \\
\hline & Total & $\begin{array}{c}177 \\
(30.2 \%)\end{array}$ & $\begin{array}{c}117 \\
(19.9 \%)\end{array}$ & $\begin{array}{c}173 \\
(29.5 \%)\end{array}$ & $\begin{array}{c}120 \\
(20.4 \%)\end{array}$ & 587 \\
\hline
\end{tabular}

Note. Percentages are computed within each row. *Sampling was done such that there is an equal sample size observed for low and high stress periods. 